\title{
Agrupamento de precipitação no estado do Pará, Brasil
}

\author{
Rainfall clustering in the state of Pará, Brazil
}

Bruna Roberta Pereira Lira ${ }^{1}$ (D), Diêgo Lima Crispim¹ ${ }^{1}$, David Figueiredo Ferreira Filho ${ }^{1}$ (D), Lindemberg Lima Fernandes ${ }^{1}$ (D) Francisco Carlos Lira Pessoa ${ }^{1}$ (D)

${ }^{1}$ Universidade Federal do Pará - UFPA, Belém, PA, Brasil. E-mails: bruna.lira@itec.ufpa.br, diego.crispim@itec.ufpa.br, davydferreira@gmail.com, lberge@ufpa.br, fclpessoa@ufpa.br

Como citar: Lira, B.R.P., Crispim, D.L., Ferreira Filho, D.F., Fernandes, L.L., \& Pessoa, F.C.L. (2020). Agrupamento de precipitação no estado do Pará, Brasil. Revista de Gestão de Água da América Latina, 17, e19. https://doi.org/10.21168/rega.v17e19

\begin{abstract}
RESUMO: Esta pesquisa tem como objetivo analisar o comportamento pluviométrico sazonal do estado do Pará em um período de 30 anos (1986-2015), através da análise de agrupamento hierárquico de Ward, por meio de dados de 108 estações pluviométricas disponibilizados pelo HidroWeb da Agência Nacional de Águas (ANA), de forma a contribuir como subsídio para gestão e planejamento dos recursos hídricos da região. Os resultados indicaram, a partir dos índices de validação, o número de formação com dois ou oito grupos, ou seja, de duas e oito regiões pluviométricas homogêneas. A avaliação em duas regiões separa o estado entre o Norte e o sul, o Norte com índices pluviométricos maiores, e o Sul com índices pluviométricos menores, com variação na sazonalidade demonstrada na normal climatológica das regiões. Já para o agrupamento em oito regiões, foram verificados diferentes padrões pluviométricos com variações na precipitação mensal média das regiões. Conclui-se que o comportamento pluviométrico no estado em sua totalidade não é homogêneo, no entanto, dentro das formações com 2 e 8 agrupamentos, o comportamento da precipitação foi semelhante, formando regiões pluviométricas homogêneas, tanto em relação a variação temporal da chuva ao longo do ano, quanto a boa coerência espacial, neste caso, podendo servir como subsídio para a gestão e o planejamento dos recursos hídricos no estado do Pará.
\end{abstract}

Palavras-chave: Gestão de Recursos Hídricos; Método de Ward; Índices de Validação; Regiões Homogêneas.

ABSTRACT: This research aims to analyze the seasonal rainfall behavior of the state of Pará over a period of 30 years (1986-2015), through Ward's hierarchical cluster analysis, using data from 108 rainfall stations provided by HidroWeb system of Agência Nacional de Águas (ANA), in order to contribute as a subsidy for the management and planning of water resources in the region. The results indicated, from the validation indexes, the number of formations with two or eight groups, that is, of two and eight homogeneous rainfall regions. The assessment in two regions separates the state between the North, with higher rainfall rates, and the South, with lower rainfall rates, with variation in seasonality demonstrated in the climatic normal of the regions. For the grouping in eight regions, different rainfall patterns were verified with variations in the average monthly precipitation of the regions. It is concluded that the pluviometric behavior in the state as a whole is not homogeneous, however, within the formations with 2 and 8 clusters, the behavior of precipitation was similar, forming homogeneous rainfall regions, both in relation to the temporal variation of rain over of the year, as to good spatial coherence, in this case, which can serve as a subsidy for the management and planning of water resources in the state of Pará.

Keywords: Water Resources Management Ward Method; Validation Indexes; Homogenous Regions.

\section{INTRODUÇÃO}

Mesmo com sua grande importância nos fenômenos climáticos do continente, a região amazônica apresenta ausência de informações em algumas de suas partes, devido a insuficiência do monitoramento de dados pluviométricos, seja pela pequena densidade de estações instaladas devido à grande área da região, ou pelo fato de muitas já se encontrarem desativadas, e outras por apresentarem erros e descontinuidades (Ishihara et al., 2013). Esse problema se deve em parte ao tamanho da região que aumenta os custos no estabelecimento e operação da rede, bem como os custos de logística e transporte de equipes técnicas para medições, coleta de dados, análise do

Recebido: Maio 27, 2020. Revisado: Agosto 01, 2020. Aceito: Setembro 11, 2020. 
comportamento das chuvas e fluxos, todos altamente cruciais para estudos hidrológicos (Gonçalves et al., 2016).

Portanto, essa variável climatológica não é quantificada em algumas áreas do território da região Amazônica, dificultando o entendimento da variação temporal e espacial da chuva. 0 conhecimento da variabilidade pluviométrica pode fornecer informações sobre o clima, as quais são essenciais para o planejamento de atividades humanas e desenvolvimento local (Amanajás \& Braga, 2012), já que os índices pluviométricos estão diretamente relacionados à disponibilidade hídrica, essencial para planejamento de sistemas de abastecimento de água, geração de energia, produções agrícolas, previsões de enchentes, medidas mitigatórias aos impactos devido à falta ou excesso de chuva (Heathman et al., 2009).

Diante destas problemáticas, diversos estudos em diferentes momentos (Amanajás \& Braga, 2012; Gonçalves et al., 2016; Azevedo et al., 2017; Neves et al., 2017; Santos et al., 2017) no meio científico, já utilizaram a análise de agrupamento ou cluster, para auxiliar na análise de chuva, principalmente em regiões com ausência ou insuficiência de dados, como é o caso da região amazônica.

A identificação de regiões homogêneas é realizada pela Análise de Agrupamentos, conhecida como análise de conglomerados, classificação ou cluster (Mingoti, 2013). É um método estatístico de análise multivariada amplamente utilizado para identificar, em grandes conjuntos de dados, entes que apresentem características similares (Pes etal., 2017). 0 método permite identificar a menor variância entre os grupos formados, associando elementos, cuja soma dos quadrados entre eles é mínima, ou ainda, que o erro desta soma seja mínimo (Santos et al., 2014; Azevedo et al., 2017; Neves et al., 2017).

0 método, detecta o menor erro interno entre os vetores que compõem cada grupo e o vetor médio do grupo. Isso equivale a identificar o menor desvio padrão entre os dados para cada grupo. Na fase inicial, o erro interno é nulo para todos os grupos, uma vez que cada vetor que compõe cada grupo é o vetor médio desse grupo. Assim, o desvio padrão para cada grupo é nulo. Na etapa seguinte, cada possibilidade de aglomeração entre os grupos 2 por 2 é verificada, e o cluster que causa o menor aumento no erro interno do grupo é escolhido (Gonçalves et al., 2016).

0 resultado do agrupamento são classificações hierárquicas, que podem ser representadas por um diagrama bidimensional, dendogramas (Menezes et al., 2015). De acordo com Pessoa (2015), a técnica interliga as amostras por associações.

As técnicas utilizadas para análise podem ser aplicadas em diversas áreas, tornando-se uma ferramenta útil para a análise de diversas situações. Segundo Menezes et al. (2015), é útil para tratamento de dados climáticos e para separar conjuntos de estações pluviométricas com características homogêneas. Conforme Amanajás \& Braga (2012), essa análise auxilia na identificação de padrões de precipitação pluvial e de regiões homogêneas de chuva, tornando-se prática habitual e desenvolvida por diferentes metodologias.

A caracterização espacial e temporais da chuva apoiam o planejamento, o estabelecimento e o gerenciamento de políticas públicas e privadas em diversas áreas (Lyra et al., 2014). 0 entendimento da precipitação através de áreas homogêneas contribui para gestão dos recursos hídricos, visto que se tornam perceptíveis os períodos e áreas de maiores ou menores índices pluviométricos. (Neves et al., 2017). Conforme Santos et al. (2017), pode indicar quais regiões são mais suscetíveis aos períodos de seca ou de chuva, bem como auxiliar na seleção de culturas agrícolas e no estabelecimento das datas de plantio mais favoráveis. Facilita a identificação das prioridades relacionadas ao planejamento de atividades que dependem do comportamento das chuvas (Menezes et al., 2015).

Neste contexto, o objetivo deste trabalho é analisar o comportamento da precipitação no estado do Pará, por meio da aplicação da técnica de agrupamento para identificação de regiões com pluviosidades homogêneas, a partir de séries temporais de precipitações mensais, que possibilitem a avaliação da variação temporal e espacial da chuva.

\section{METODOLOGIA}

\section{ÁREA DE ESTUDO}

O presente estudo abrange o estado do Pará (Figura 1) na região Norte do Brasil, e inserido na área em que está compreendida a Amazônia Brasileira, sendo o segundo maior estado em extensão territorial do país (Instituto Brasileiro de Geografia e Estatística, 2010). 


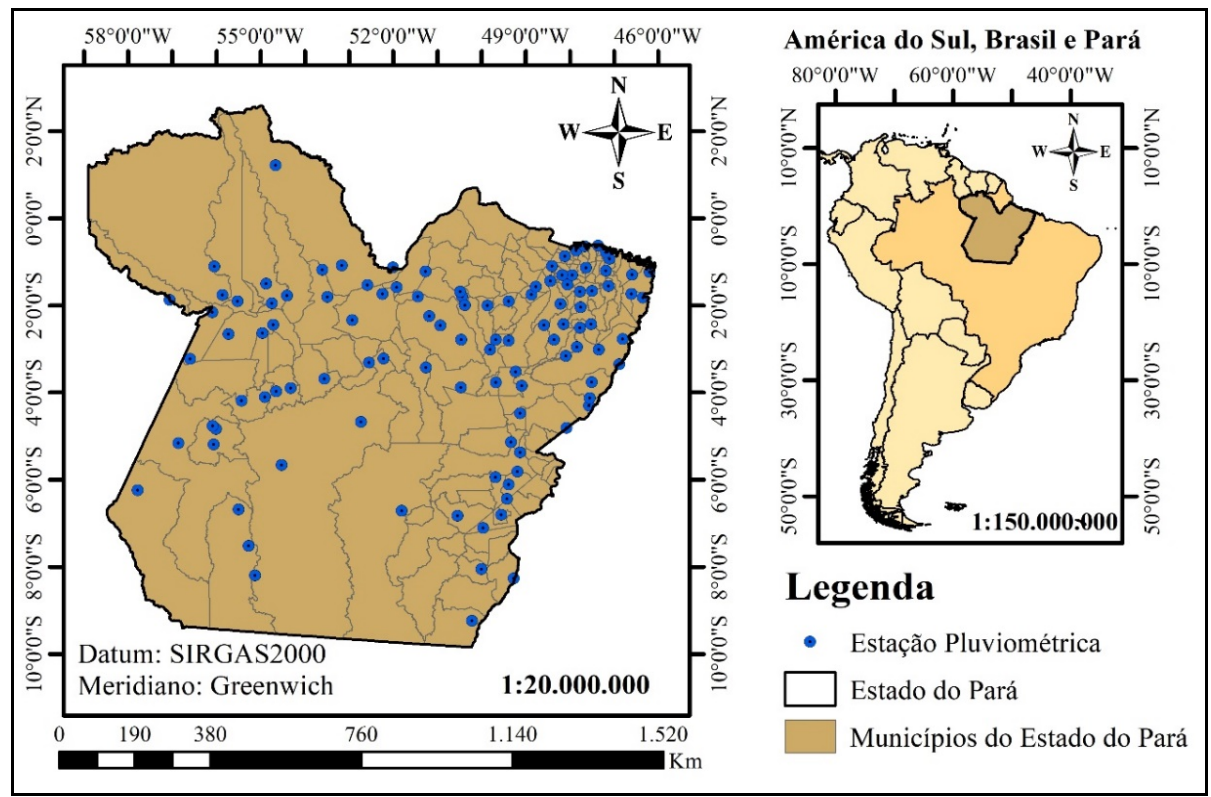

Figura 1 - Mapa de localização da área de estudo, o estado brasileiro do Pará, juntamente com a espacialização das estações pluviométricas utilizadas para análise de agrupamento

Fonte: Elaborado pelos Autores, com a fonte de dados da Agência Nacional de Águas (2020) e IBGE (Instituto Brasileiro de Geografia e Estatística, 2020)

O estado do Pará é caracterizado pela alta variabilidade da pluviosidade, em função de diferentes sistemas atmosféricos que atuam sobre a região (Menezes et al., 2015). De acordo com a classificação de Köppen a região pertence ao clima do tipo "A", designado a climas tropicais com altos índices pluviométricos e com temperaturas médias maiores que $18{ }^{\circ} \mathrm{C}$, predominantemente quente e úmido. Conforme Ferreira Filho et al. (2020) as médias de chuvas anuais estão próximas dos $2.000 \mathrm{~mm}$, variando entre $1.600 \mathrm{~mm}$ e $1900 \mathrm{~mm}$, e as médias de temperatura do ar anuais variam entre 24 e 26으, com umidade relativa do ar de aproximadamente $80 \%$.

\section{COLETA E ANÁLISE DOS DADOS}

No portal do HidroWeb da Agência Nacional de Águas (2020), foram verificadas as estações pluviométricas que estivessem no território do estado do Pará e com séries históricas que tivessem no mínimo 20 anos de dados no período de estudo de 30 anos, de janeiro de 1986 a dezembro de 2015. Algumas séries de dados apresentaram falhas, porém, optou-se pela não correções das falhas, conforme realizado por Gomes \& Fernandes (2019), pois se trata de uma região com baixa densidade de informações. Portanto, selecionou-se 108 estações com os códigos descritos. A partir das séries históricas destas estações, foram calculadas as médias mensais do período de estudo para cada estação, demonstrando o comportamento sazonal da precipitação.

\section{ANÁLISE DE AGRUPAMENTO}

Os procedimentos para aplicação e análise de agrupamento ocorrem em quatro etapas: a escolha das variáveis classificatórias, especificação da medida de similaridade, seleção do método de agrupamento e decisão quanto ao número de grupos a serem formados (Menezes et al., 2015). Neste, a variável é a precipitação média mensal, a medida de similaridade é a distância euclidiana e o método de agrupamento é o Hierárquico de Ward. Conforme Santos et al. (2014), a distância euclidiana (Equação 1) é uma das medidas para regionalização de dados hidrológicos e climáticos.

$D_{i j}=\sqrt{\sum_{k=1}^{n}\left(X_{k i}-X_{k j}\right)^{2}}$

Onde: $D_{i j}$ é a distância entre os elementos observados i e j; o $X_{k i}$ é o valor assumido pela variável $K$ para observação i; e o $X_{k j}$ o valor da variável ${ }_{k}$ para observação j; 
O método foi proposto por Ward Junior (1963), consiste no agrupamento hierárquico no qual a medida de similaridade é usada para determinar a menor variação entre os dados dos grupos. Calculase a soma dos quadrados Equação 2 passo a passo (Pessoa, 2015).

$S S_{i}=\sum_{k=1}^{n i}\left(X_{i j}-\mu_{i}\right)^{\prime \prime}\left(X_{i j}-\mu_{i}\right)$

onde $_{n i}$ é o número de elementos no conglomerado, quando se está no passo $k$ do processo de agrupamento; o $X_{i j}$ é o vetor de observações do j-ésimo elemento amostral que pertence ao i-ésimo conglomerado; o $\mu_{i}$ é o centróide do conglomerado; e o $S S_{i}$ representa a soma dos quadrados correspondente ao conglomerado.

No passo $K$, a soma de quadrados total dentro dos grupos é definida pela Equação 3.

$S S R=\sum_{i=1}^{g k} S S_{i}$

em que $g k$ é o número de grupos existentes quando se está no passo $K$.

A distância entre os conglomerados $C_{i}$ e $C_{i}$ é definida na Equação 4:

$d(C i, C i)=\left[\frac{n i n i}{n i+n i}\right]\left(\mu_{i}-\mu_{i}\right)^{\prime}\left(\mu_{i}-\mu_{i}\right)$

Em cada passo do algoritmo de agrupamento, os dois conglomerados $\left(C_{i}\right.$ e $\left.C_{i}\right)$ que minimizam a distância são combinados. As comparações de conglomerados, que tem tamanhos diferentes, sofrem uma penalização representada pelo fator de ponderação $\left[\frac{n i n i}{n i+n i}\right]$. Quanto maior for os valores de nienié a discrepância entre eles, maior foi o valor do fator de penalização, aumentando a distância entre os centroides dos conglomerados comparados (Pessoa, 2015).

Na Figura 2, tem-se as etapas do algoritmo do método de agrupamento hierárquico de Ward, para delimitação de regiões hidrológicas homogêneas.

1. Iniciar operando a matriz de similaridade, considerando cada elemento como um único conglomerado;

2. Calcular o valor da soma dos quadrados SSi para cada um dos possíveis pares de conglomerados segundo a Equação (2);

3. Calcular a soma de quadrados total dentro dos grupos, Equação (3);

4. Calcular a distância entre os conglomerados por meio da Equação (4);

5. Repetir os passos anteriores n - vezes, de maneira que todos os n elementos pertençam a um grupo ao fim do algoritmo.

Figura 2 - Algoritmo do método hierárquico de Ward Fonte: Adaptado de Pessoa (2015)

\section{ÍNDICES DE VALIDAÇ̃̃o}

Para diminuir possíveis questionamentos concernentes as subjetividades na seleção de agrupamentos iniciais foram aplicadas índices de validação com a finalidade de analisar os resultados originados pelos algoritmos de agrupamento (Halkidi et al., 2002).

Deste modo, as técnicas de avaliação dos resultados na formação dos grupos possuem quatro características fundamentais: definir se existe uma estrutura não aleatória nos dados com a finalidade de impedir tendência nos resultados; estabelecer a quantidade de grupos iniciais; analisar como um resultado de Clustering se estrutura a um determinado conjunto de dados, sendo essa a única informação disponível; e analisar o quão bem situados estão as observações dentro dos grupos baseados nas partições alcançadas conforme outras fontes de dados.

Nesse estudo foram empregadas as validações por meio dos índices da Tabela 1, que são Davies Bouldin (DB), Silhouette (SIL), PBM e Xie Beni (XB). Todos os índices de validações foram aplicados no software livre R. 
Tabela 1 - Índices de validação para definição do número inicial de agrupamentos

\begin{tabular}{|c|c|c|c|}
\hline Índice de validação & Equação & & Fonte \\
\hline Davies Bouldin (DB) & $D B=\frac{1}{K} \sum_{i=1}^{K} R i, q t$ & (1) & Pakhira et al. (2004) \\
\hline Silhouette (SIL) & $\bar{s}(k)=\frac{1}{n} \sum_{i=1}^{n} s(i)$ & (2) & Silva et al. (2018) \\
\hline PBM & $P B M(k)=\left(\frac{1}{k} * \frac{E 1}{E k} * D k\right)^{2}$ & (3) & Pakhira et al. (2004) \\
\hline Xie Beni (XB) & $J m(U, Z)=\sum_{j=1}^{n} \sum_{k=1}^{k}(U k j)\|x j-z k\|^{2}$ & (4) & Pakhira et al. (2004) \\
\hline
\end{tabular}

Fonte: Adaptado de Pakhira et al. (2004), Parchure \& Gedam (2019).

\section{RESULTADOS E DISCUSSÃO}

Primeiramente foi determinado o número de grupos, a partir dos índices de validação, que apresentam particularidades distintas, no qual uns diminuem o seu coeficiente para obter um melhor resultado de agrupamento como, por exemplo, os métodos Davies Bouldin (DB) e Xie Beni (XB), enquanto os outros potencializam o resultado do coeficiente para alcançar a melhor formação dos grupos, a título de exemplo, Silhouette e PBM (Pakhira et al., 2004; Parchure \& Gedam, 2019). Estes apresentaram a composição de dois distintos agrupamentos iniciais, determinados pelos índices Davies Bouldin (DB), Silhouette (SIL) e PBM, enquanto o índice de validação Xie Beni (XB) estabeleceu que o número ideal de grupos são 8, conforme o apresentado na Tabela 2. Deste modo, nesse estudo, considerou-se essas duas proposições de agrupamentos iniciais apresentados pelos índices.).

Tabela 2 - Determinação do número ideal de agrupamentos por meio de índices de validação

\begin{tabular}{l|c|c|c|c|c|c|c|c|c|c}
\hline \multicolumn{1}{c}{ Número do Grupo } & $\mathbf{2}$ & $\mathbf{3}$ & $\mathbf{4}$ & $\mathbf{5}$ & $\mathbf{6}$ & $\mathbf{7}$ & $\mathbf{8}$ & $\mathbf{9}$ & $\mathbf{1 0}$ \\
\hline Davies Bouldin (DB) & 1,056 & 1,334 & 1,236 & 1,182 & 1,104 & 1,058 & 1,094 & 1,227 & 1,207 \\
\hline Silhouette (SIL) & 0,371 & 0,241 & 0,238 & 0,243 & 0,297 & 0,293 & 0,282 & 0,27 & 0,26 \\
\hline PBM & 0,947 & 0,681 & 0,775 & 0,705 & 0,872 & 0,738 & 0,678 & 0,586 & 0,523 \\
\hline Xie Beni (XB) & 7,014 & 8,346 & 6,485 & 5,239 & 4,327 & 3,763 & 3,329 & 4,988 & 4,622 \\
\hline
\end{tabular}

Fonte: Dados da pesquisa (2020).

Em seguida, foi aplicado o método hierárquico Aglomerativo de Ward às precipitações médias mensais das 108 estações pluviométricas do estado do Pará, usando como medida de dissimilaridade a distância Euclidiana. Assim, teve-se como resultado o dendograma apresentado na Figura 3, o primeiro corte subdividiu o estado em duas grandes regiões pluviométricas sazonais homogêneas, denominadas de $1 \mathrm{~A}$ e $2^{\mathrm{a}}$, como foi sugerido pelo índice de validação. Entretanto, se opta-se por um número maior de grupos, bastava fazer um corte em uma distância menor, assim foi feito pra subdivisão em oito regiões.

As estações pluviométricas das duas regiões homogêneas quanto à pluviosidade sazonal foram distribuídas no estado do Pará (Figura 4), juntamente com o padrão pluviométrico para a cada região. De acordo com Azevedo et al. (2017), no período chuvoso o oceano Pacífico modula a precipitação sobre a região da Amazônia oriental, enquanto no período menos chuvoso é o oceano Atlântico.

A região 1A (vermelho) foi constituída por 67 estações pluviométricas, representa $62,04 \%$ do total, estão localizadas no Norte do estado, onde estão o litoral nordeste paraense e a Região Metropolitana de Belém (RMB). Nesta, foram verificados os maiores índices pluviométricos, com semestre mais chuvoso em janeiro (281,9 mm), fevereiro (329,7 mm), março (409,6 mm), abril $(382,8 \mathrm{~mm})$, maio $(286,7 \mathrm{~mm})$ e junho (164,3 mm), enquanto o semestre menos chuvoso foi julho (123 mm), agosto $(71,8 \mathrm{~mm})$, setembro $(52,2 \mathrm{~mm})$, outubro $(54,4 \mathrm{~mm})$, novembro $(68,7 \mathrm{~mm})$ e dezembro $(136,1 \mathrm{~mm})$.

A região 2A foi composta por 41 estações, correspondendo a 37,96\% do total, onde estão localizadas majoritariamente nas regiões sudeste e sudoeste do estado. Esta apresentou pluviosidade menor e se verificou o semestre mais chuvoso em dezembro $(180,79 \mathrm{~mm})$, janeiro $(245,7 \mathrm{~mm})$, fevereiro $(267,6 \mathrm{~mm})$, março $(317,8 \mathrm{~mm})$, abril $(254,4 \mathrm{~mm})$ e maio $(157,52 \mathrm{~mm})$ enquanto o semestre menos chuvoso foi em junho $(58,2 \mathrm{~mm})$, julho $(36,4 \mathrm{~mm})$, agosto $(31,4 \mathrm{~mm})$, setembro $(57,8 \mathrm{~mm})$, outubro $(98,6 \mathrm{~mm})$ e novembro $(132,6 \mathrm{~mm})$. 


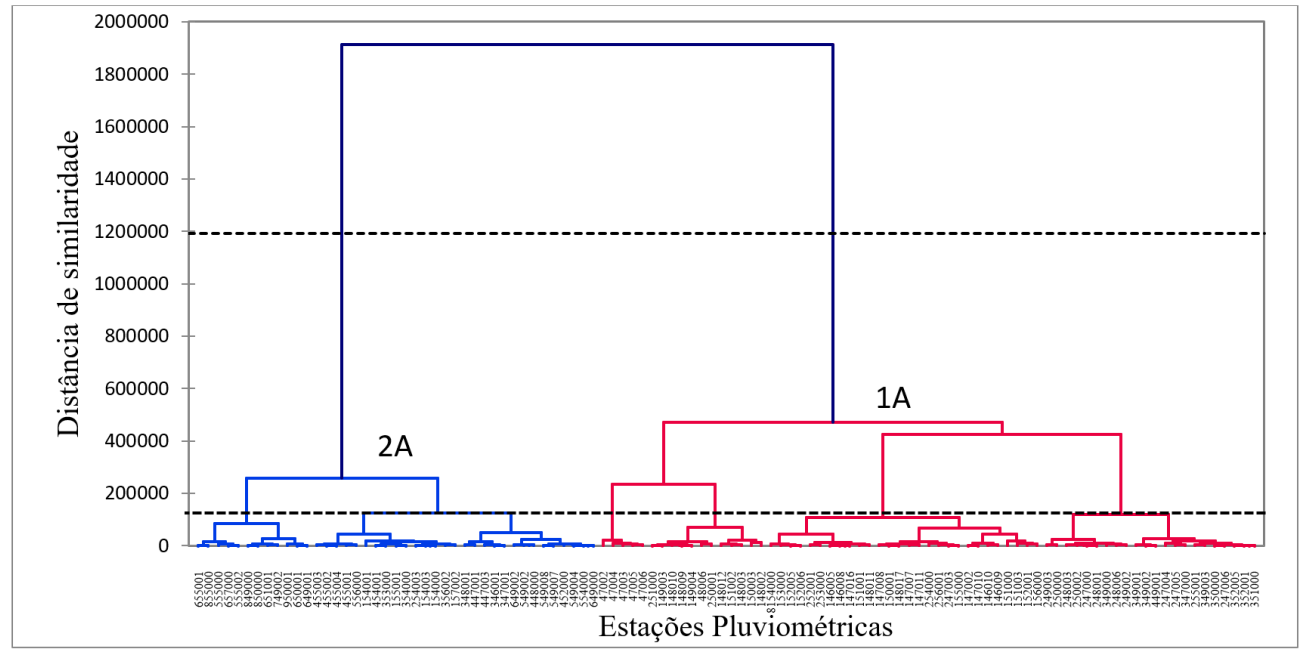

Figura 3 - Dendograma de divisão do estado do Pará em diferentes regiões com pluviosidade com variabilidades semelhantes.

Fonte: Autores (2020)

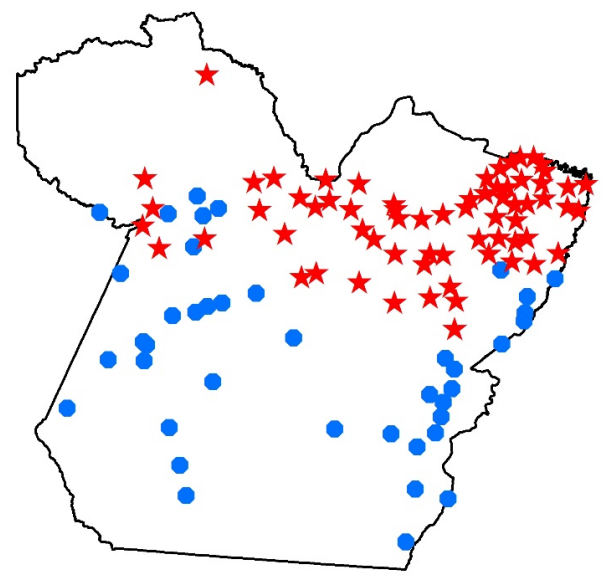<smiles></smiles>

Regiôes Homogêneas

$\star 1 \mathrm{~A}-$ Norte

- $2 \mathrm{~A}-\mathrm{Sul}$

1:18.000.000

Datum: SIRGAS2000

Meridiano: Greenwich
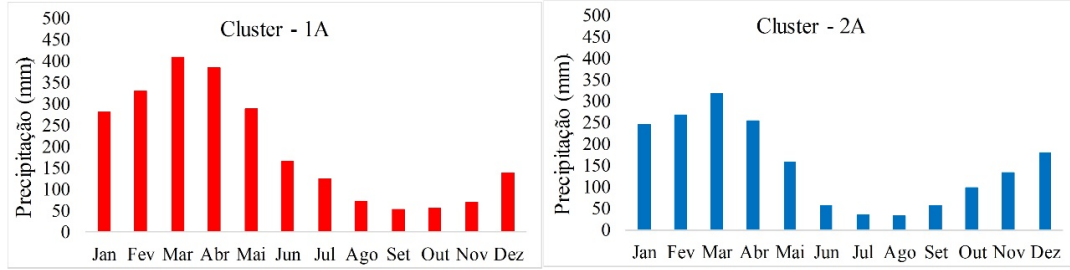

Figura 4 - Regiões homogêneas quanto à pluviosidade médias mensal no estado do Pará, para divisão em dois grupos

Fonte: Autores (2020)

O resultado obtido neste estudo corrobora com o apresentado por Moraes et al. (2005), que indicaram que as maiores precipitações anuais ocorrem na porção norte do Estado, enquanto à Sudeste ocorrem os menores totais. No trabalho de Ishihara et al. (2014), em que foi avaliada a precipitação na Amazônia Legal, durante 1978 a 2007, se constatou que as maiores taxas de precipitação ocorrem no extremo norte da região, principalmente no Amapá, litoral do Pará e Noroeste do Amazonas, o que corrobora com o obtido na presente avaliação. Os altos índices pluviométricos desta região estão relacionados aos padrões oceano-atmosfera e as anomalias de temperatura da superfície do mar. Conforme Menezes et al. (2015), a ZCIT é um dos principais responsáveis pela chuva na região.

Segundo Santos et al. (2017), é importante salientar que os eventos secos e chuvosos nesta área estão associados, principalmente, a variabilidade climática dos oceanos, Atlântico (Gradiente do Atlântico) e Pacífico (ENOS). Além de que, de acordo com Gonçalves et al. (2016), existe uma tendência de aumento e diminuição da precipitação, durante os anos de ocorrência de eventos de La Niña e El Niño, respectivamente. 
Já o número de regiões homogêneas encontradas para o estado do Pará, neste estudo, é diferente dos apresentados por Menezes et al. (2015), que definiram três regiões pluviométricas homogêneas para o estado, utilizadas médias mensais de precipitação de 66 estações, para o período de 1982 a 2011; e por Santos et al. (2017), que identificaram três Regiões homogêneas para o estado através do índice de precipitação normalizada, são: a RH-1 no nordeste, com 26 estações; a RH-2 no centro e noroeste, com 32 estações; e a RH-3 no Sul, com 14 estações. A diferença entre os resultados analisados está na definição de número de grupos a serem formados, pois neste estudo aplicou-se vários índices de validações, diferente dos demais autores citados acima.

0 índice de validação Xie Beni estabeleceu o número 8 como ideal para a formação dos grupos. Deste modo, realizou-se outro corte no dendograma para subdividiu o estado do Pará em oito regiões de pluviosidade sazonal homogênea. A espacialização das estações pluviométricas das oito regiões está distribuída na Figura 5, juntamente com o ciclo anual da precipitação de cada região homogênea, que apresenta os regimes pluviométricos sazonais, por meio dos valores médios de precipitações mensais. Nota-se que onde se localizava a região 1A foi definido um novo cenário que subdividiu esta região em $1 \mathrm{~B}, 2 \mathrm{~B}, 3 \mathrm{~B}, 5 \mathrm{~B}$ e $8 \mathrm{~B}$, enquanto a região $\mathbf{2 A}$, no Sul, apresentou o cenário 4B, 6B e 7B. Destaca-se que as regiões de pluviosidade com variabilidade semelhante para formação com dois ou oito grupos apresentaram comportamento similar quanto a sazonalidade, visto que ambas possuem dois períodos bem definidos de uma estação chuvosa e outra menos chuvosa, diferenciando-se apenas quanto aos meses inseridos nesses dois períodos.

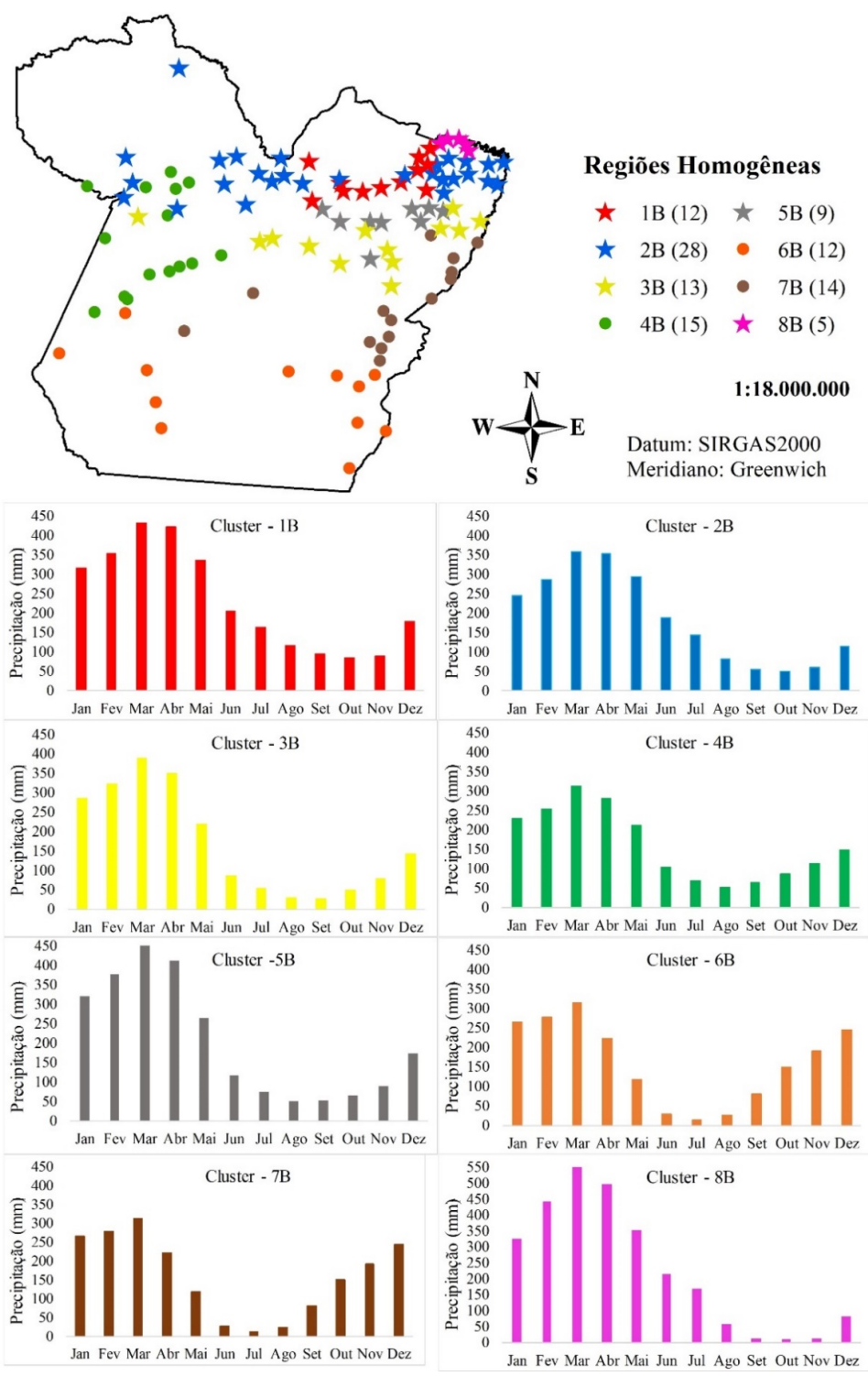

Figura 5 - Regiões homogêneas quanto à pluviosidade médias mensal no estado do Pará, para divisão em oito grupos Fonte: Autores (2020) 
Nota-se que o comportamento pluviométrico sazonal das Regiões 1B, 2B, 3B e 5B apresentam semelhança na distribuição pluviométrica durante o ano, porém com diferença quanto ao volume precipitado. De acordo com Menezes et al. (2015), o descolamento da precipitação é influenciado pela Zona de Convergência Intertropical - ZCIT, com aumento da precipitação a partir do mês de dezembro e fim da estação chuvosa em maio.

Destaca-se que os estudos de Menezes et al. (2015) e Amanajás \& Braga (2012) apresentaram o mês de março como o mais chuvoso em todas as regiões homogêneas quanto à pluviosidade formadas, o que corrobora com os resultados deste estudo. Os sistemas meteorológicos se diferem ao longo do ano e de forma espacial, como pode ser observado pela ocorrência da ZCIT que exerce um papel importante e principal na modulação do regime de chuva na região equatorial (Lopes et al., 2013). Além da influência dos fenômenos climáticos tropicais de larga escala de El Niño e La Niña (Souza et al., 2017) e as fases do dipolo de temperatura da superfície do mar (TSM) do oceano Atlântico (Azevedo et al., 2017).

A Região 1B composta por 12 estações pluviométricas (11,1\%), indicadas pela cor vermelha, localizadas no nordeste paraense em áreas as margens do rio Pará, apresentou o semestre mais chuvoso em janeiro $(315,6 \mathrm{~mm})$, fevereiro $(353,9 \mathrm{~mm})$, março $(431,4 \mathrm{~mm})$, abril $(422,6 \mathrm{~mm})$, maio $(336,2 \mathrm{~mm})$ e junho (206,0 mm), enquanto o semestre menos chuvoso é em julho (163,8 mm), agosto (115,8 mm), setembro $(93,4 \mathrm{~mm})$, outubro $(84,9 \mathrm{~mm})$, novembro $(88,5 \mathrm{~mm})$ e dezembro $(177,3 \mathrm{~mm})$. A Região 2 B formada por 28 estações pluviométricas (25,9\%), representadas pela cor azul, espalhadas no norte do estado, teve o semestre mais chuvoso semelhante ao anterior, porém, os índices são diferentes, em janeiro (244,3 mm), fevereiro $(285,4 \mathrm{~mm})$, março $(357,1 \mathrm{~mm})$, abril $(351,9 \mathrm{~mm})$, maio $(293,5 \mathrm{~mm})$ e junho $(189,2 \mathrm{~mm})$, enquanto o semestre menos chuvoso em julho $(144,3 \mathrm{~mm})$, agosto $(81,8 \mathrm{~mm})$, setembro $(54,8 \mathrm{~mm})$, outubro $(51,3 \mathrm{~mm})$, novembro $(60,7 \mathrm{~mm})$ e dezembro $(113,2 \mathrm{~mm})$. A variabilidade da precipitação entre os dois períodos (chuvoso e menos chuvoso) mostra a influência dos fenômenos atmosféricos na variação da precipitação (Campos etal., 2015). Entre os principais sistemas atmosférico atuantes nestas regiões, a Zona de Convergência Intertropical (ZCIT), brisa marítima e as linhas de instabilidades - LI (Moraes et al., 2005; Campos et al., 2015).

A Região 3B constituída por 13 estações pluviométricas (12,0\%), indicadas pela cor amarela, distribuídas no centro do estado, apresentou o semestre chuvoso em dezembro (142,6 mm), janeiro $(284,1 \mathrm{~mm})$, fevereiro $(322,6 \mathrm{~mm})$, março $(387,6 \mathrm{~mm})$, abril $(348,9 \mathrm{~mm})$ e maio $(219,6 \mathrm{~mm})$ e o semestre menos chuvoso em junho (84,7 mm), julho (54,1 mm), agosto $(28,3 \mathrm{~mm})$, setembro $(27,1 \mathrm{~mm})$, outubro (47,4 mm) e novembro (78,2 mm); A Região 4B com 15 estações pluviométricas $(13,9 \%)$, na cor verde, localizadas no centro-oeste do estado, região de fronteira com o estado do Amazonas, apresentou o semestre chuvoso em dezembro (148 mm), janeiro (228,8 mm), fevereiro (253,8 mm), março (312,6 mm), abril $(281,8 \mathrm{~mm})$ e maio $(210,8 \mathrm{~mm})$ enquanto o semestre menos chuvoso foi em junho $(103,5 \mathrm{~mm})$, julho $(70,0 \mathrm{~mm})$, agosto $(50,3 \mathrm{~mm})$, setembro $(63,1 \mathrm{~mm})$, outubro $(86,2 \mathrm{~mm})$ e novembro $(112,9 \mathrm{~mm})$. De acordo com Dias et al. (2020), existe a transição sazonal com aumento da precipitação a partir de dezembro, além de um período menos chuvoso de junho a novembro, isto ocorre porque nesse período a região sofre influência da migração sazonal da ZCIT para o hemisfério norte.

A Região 5B composta por 9 estações pluviométricas (8,3\%), de cor cinza, localizadas no nordeste paraense, que teve o semestre mais chuvoso em dezembro $(174,1 \mathrm{~mm})$, janeiro $(320,4 \mathrm{~mm})$, fevereiro (377,1 mm), março (478,3 $\mathrm{mm})$, abril $(412,1 \mathrm{~mm})$ e em maio $(263,7 \mathrm{~mm})$ e o semestre menos chuvoso em junho $(115,9 \mathrm{~mm})$, julho $(74,6 \mathrm{~mm})$, agosto $(51,0 \mathrm{~mm})$, setembro $(51,3 \mathrm{~mm})$, outubro $(63,2 \mathrm{~mm})$ e novembro $(90,4 \mathrm{~mm})$;

A Região 6B foi constituída por 12 estações pluviométricas (11,1\%), indicadas pela cor laranja, localizadas ao sul paraense, caracterizam-se pelo início do período chuvoso em novembro e o fim em abril, teve o semestre mais chuvoso em novembro $(191,4 \mathrm{~mm})$, dezembro $(244,1 \mathrm{~mm})$, janeiro $(265,6 \mathrm{~mm})$, fevereiro $(278,3 \mathrm{~mm})$, março $(313,2 \mathrm{~mm})$ e abril $(222,4 \mathrm{~mm})$ e o semestre menos chuvoso em maio (117,1 mm), junho $(27,5 \mathrm{~mm})$, julho $(13,2 \mathrm{~mm})$, agosto $(25,4 \mathrm{~mm})$, setembro $(80,9 \mathrm{~mm}) \mathrm{e}$ outubro (149,0 mm). Oliveira Vieira et al. (2013) propuseram que a Zona de Convergência do Atlântico Sul - ZCAS é um dos responsáveis pela ocorrência de chuva no sul do estado do Pará. Segundo Lopes et al. (2013), a convecção organizada pela Alta da Bolívia (AB), que surge em decorrência do forte aquecimento no altiplano boliviano, também influencia na precipitação desta região.

A Região 7B que agrupou 14 estações pluviométricas (13,0\%), representadas pela cor marrom, localizadas no centro-leste, apresentou o semestre mais chuvoso em dezembro (161,7 mm), janeiro (246,9 mm), fevereiro (273,4 mm), março (327,6 mm), abril (253,4 mm) e maio $(135,1 \mathrm{~mm})$ e o semestre menos chuvoso em junho $(36,0 \mathrm{~mm})$, julho $(20,3 \mathrm{~mm})$, agosto $(16,4 \mathrm{~mm})$, setembro $(32,4 \mathrm{~mm})$, outubro $(68,7 \mathrm{~mm})$ e novembro $(103,5 \mathrm{~mm})$. 
A Região 8B apresentou comportamento diferente e as maiores variações de precipitação total média entre os períodos mais e menos chuvosos. Formada por 5 estações pluviométricas $(4,6 \%)$, indicadas pela cor rosa, localizadas no nordeste paraense na zona costeira do estado, conhecida como microrregião do salgado, teve o semestre mais chuvoso para janeiro $(326,0 \mathrm{~mm})$, fevereiro $(442,3$ $\mathrm{mm})$, março $(587,4 \mathrm{~mm})$, abril $(496,6 \mathrm{~mm})$, maio $(351,5 \mathrm{~mm})$ e junho $(213,9 \mathrm{~mm})$, enquanto o semestre menos chuvoso é em julho $(169,8 \mathrm{~mm})$, agosto $(57,9 \mathrm{~mm})$, setembro $(13,2 \mathrm{~mm})$, outubro $(10,8 \mathrm{~mm})$, novembro $(12,3 \mathrm{~mm})$ e dezembro $(81,4 \mathrm{~mm})$. Este comportamento tem relação com os fenômenos climáticos, principalmente quando as anomalias do pacífico visto que o Dipolo positivo em paralelo com El Niño tende a reduzir a precipitação, enquanto o dipolo negativo com La Niña intensifica as mesmas (Gomes \& Fernandes, 2019).

E importante destacar que a divisão em duas regiões enfatiza a diferença do comportamento pluviométrico, principalmente no volume precipitado ao longo do ano, demostrando que o comportamento é heterogêneo dentro do estado do Pará. Enquanto na divisão em oito regiões os volumes precipitados são mais similares entre si, coerente para o uso na gestão e planejamento.

\section{CONCLUSÃo}

Não existe homogeneidade no comportamento pluviométrico sazonal em sua totalidade no estado. Contudo, com o agrupamento da precipitação média mensal observou-se que algumas estações apresentam comportamento semelhante, formando regiões pluviométricas homogêneas.

Por meio da identificação de regiões homogêneas foi possível verificar comportamentos pluviométricos semelhantes, tanto em relação à similaridade da variabilidade temporal da chuva ao longo do ano, como a boa coerência espacial, estando as estações relativamente próximas. coincidindo com os principais sistemas atmosféricos atuantes na região. Independentemente dos números de formação, com dois ou oito grupos indicados pelos índices de validação, em ambos os casos são evidenciadas duas estações chuvosas bem definidas quanto ao total precipitado. As essas informações apresentadas podem contribuir como subsídio para a gestão e o planejamento dos recursos hídricos a divisão em duas regiões é possível ver a diferença pluviométrica entre norte e sul no estado do Pará, enquanto em oito regiões temos comportamento pluviométricos mais específicos principalmente nas regiões que apresentam ausência ou insuficiência de dados.

\section{REFERÊNCIAS}

Agência Nacional de Águas - ANA. (2020). Brasília: ANA. Recuperado em 12 de maio de 2020, de https://www.ana.gov.br/

Amanajás, J. C., \& Braga, C. C. (2012). Padrões espaço-temporal pluviométricos na Amazônia Oriental utilizando análise multivariada. Revista Brasileira de Meteorologia, 27(4), 423-434.

Azevedo, F. T. M., Souza, E. B., Franco, V. S., \& Souza, P. F. S. (2017). Prognóstico sazonal de precipitação regionalizada na Amazônia Oriental. Revista Brasileira de Geografia Física, 10(5).

Campos, T. L. O. B., Mota, M. A. S., \& Santos, S. R. Q. (2015). Eventos extremos de precipitação em Belém-PA: uma revisão de notícias históricas de jornais. Revista Ambiente \& Água, 10(1), 182-194.

Dias, G., Gomes, D., Serrão, E., Lima, A., Miranda, S., Júnior, J., \& Souza, A. (2020). Associação entre os Eventos de Enos e a distribuição Espaço-Temporal da Precipitação na Bacia Hidrográfica do Rio Capim (Ma-Pa). Revista Brasileira de Climatologia, 26.

Ferreira Filho, D., Lira, B., Crispim, D., Pessoa, F., \& Fernandes, L. (2020). Análise pluviométrica no estado do pará: comparação entre dados obtidos de estações pluviométricas e do satélite GPCC. Revista Brasileira de Climatologia, 26, http://dx.doi.org/10.5380/abclima.v26i0.67914

Gomes, A. B. B., \& Fernandes, L. F. (2019). Estudo do Regime Pluviométrico da Microrregião do Salgado no Estado do Pará. Revista AIDIS de Ingeniería y Ciencias Ambientales, 12(2), http://dx.doi.org/10.22201/iingen.0718378xe.2019.12.2.62023

Gonçalves, M. F., Blanco, C. J. C., Dos Santos, V. C., Oliveira, L. L. S., \& Pessoa, F. C. L. (2016). Identification of Rainfall Homogenous Regions taking into account El Niño and La Niña and Rainfall Decrease in the state of Pará, Brazilian Amazon. Acta Scientiarum, 38(2), 209-216.

Halkidi, M., Batistakis, Y., \& Vargiannis, M. (2002). Cluster validity methods: Part. I. SIGMOD Record, 31(2), 40-45.

Gary C. Heathman; Myriam Larose; Michael H. Cosh; Rajat Bindlishc (2009). Surface and profile soil moisture spatio-temporal analysis during an excessive rainfall period in the Southern Great Plains, USA. Catena, 78(2), 159-169.

Instituto Brasileiro de Geografia e Estatística - IBGE. (2010). Recuperado em 12 de maio de 2020, de https://cidades.ibge.gov.br/brasil/pa/panorama 
Instituto Brasileiro de Geografia e Estatística - IBGE. (2020). Cidades e estados: Belém. Rio de Janeiro: IBGE. Recuperado em 12 de maio de 2020, de https://www.ibge.gov.br/cidades-e-estados/pa/belem.html

Ishihara, J. H., Fernandes, L. F., Duarte, A. A. M., \& Loureiro, G. E. (2013). Avaliação do monitoramento pluviométrico da Amazônia legal. Revista Engenharia Ambiental: Pesquisa e Tecnologia, 10(3), 132-143.

Ishihara, J. H., Fernandes, L. L., Duarte, A. A. M., Duarte, A. R. C. L. M., Ponte, M.X., \& Loureiro, G. E. (2014). Quantitative and spatial assessment of precipitation in the Brazilian Amazon (Legal Amazon) - (1978 to 2007). Revista Brasileira de Recursos Hídricos - RBRH,19(1), 29-39.

Lopes, M. N. G., Souza, E. B., \& Ferreira, D. B. S. (2013). Climatologia regional da precipitação no estado do Pará. Revista Brasileira de Climatologia, 12, 84-102.

Lyra, G. B., Oliveira-Júnior,J. F., \&Zeri, M. (2014). Cluster analysis applied to the spatial and temporal variability of monthly rainfall in Alagoas state, Northeast of Brazil. International Journal of Climatology, 34(13), 3546-3558.

Menezes, F. P., Fernandes, L. L., \& Rocha, E. J. P. (2015). O Uso da Estatística para Regionalização da Precipitação no Estado do Pará, Brasil. Revista Brasileira de Climatologia, 16.

Mingoti, S. A. (2013). Análise de dados a través de métodos de estatística multivariada: uma abordagem aplicada. Belo Horizonte: Editora UFMG. p. 295.

Moraes, B. C., Costa, J. M. N., Costa, A. C. L., \& Costa, M. H. (2005). Variação espacial e temporal da precipitação no Estado do Pará. Acta Amazonica, 35(2)

Neves, R. R., Gonçalves, E. D., Pessoa, F. C. L., Fernandes, L. L., Gómez, Y. D., \& Santos, J. I. N. (2017). Identificação de regiões pluviometricamente homogêneas na sub bacia Trombetas. Revista AIDIS de Ingeniería y Ciencias Ambientales, 10(2), 125135.

Oliveira Vieira, S., Satyamurty, P. E., \& Andreoli, R. V. (2013). On the South Atlantic Convergence Zone affecting southern Amazonia in austral summer. Atmospheric Science Letters, 14, 1-6.

Pakhira, M., Bandyopadhyay, S., \& Maulik, U. (2004). Validity index for crisp and fuzzy clusters. Pattern Recognition, 37, 487-501.

Parchure, A. S., \& Gedam, S. K. (2019). Homogeneous regionalization via L-moments for Mumbai City, India. Meteorology hydrology and water management. Research and Operational Applications, 7(2), 73-83.

Pes, M. P., Pereira, E. B., Marengo, J. A., Martins, F. R, Heinemann, D., \& Schmidt, M. (2017). Climate trends on the extreme winds in Brazil. Renewable Energy, 109, 110-120.

Pessoa, F. C. L. (2015). Desenvolvimento de uma metodologia para regionalização de curvas de permanência de vazões da Amazônia (Tese de doutorado). Universidade Federal do Pará, Belém. Recuperado em 15 de maio de 2020, de http://proderna.propesp.ufpa.br/ARQUIVOS/teses/Francisco.pdf

Santos, E. B., Lucio, P. S., \& Silva, C. M. S. (2014). Precipitation regionalization of the Brazilian Amazon. Atmospheric Science Letters, 192, 185-192.http://dx.doi.org/10.1002/asl2.535.

Santos, S. R. Q., Braga, C. C., Sansigolo, C. A., \& Santos, A. P. P. (2017). Determinação de regiões homogêneas do Índice de precipitação normalizada (SPI) na Amazônia oriental. Revista Brasileira de Meteorologia, 32(1), 111-122.

Silva, M. N. A., Pessoa, F. C. L., Silveira, R. N. P. O., Rocha, G. S., \& Mesquita, D. A. (2018). Determinação da homogeneidade e tendência das precipitações na bacia Hidrográfica do Rio Tapajós. Revista Brasileira de Meteorologia, 33, 665-675.

Souza, E. B., Ferreira, D. B. S., Guimarães, J. T. F., Franco, V. S., Azevedo, F. T. M., Moraes, B. C., \& Souza, P.J. O. P. (2017). Padrões climatológicos etendências da precipitação nos regimes chuvoso e seco na Amazônia Oriental. Revista Brasileira de Climatologia, 13(21), 81-93.

Ward Junior, J.H. (1963). Hierarchical grouping to optimize an objective function. Journal of the American Statistical Association, $58,236-244$. 